\title{
EVALUACIÓN COMPARATIVA DEL RENDIMIENTO EN LA OBTENCIÓN DE ALCALOIDES TOTALES PARA LOS FRUTOS VERDES Y ROJOS DE Solanum pseudocapsicum L.
}

\section{COMPARATIVE EVALUATION OF TOTAL ALKALOIDS YIELD FOR GREEN AND RED FRUITS OF Solanum pseudocapsicum L.}

\author{
Luis F. Mejía ${ }^{1}$; Rigoberto Gómez ${ }^{2}$ \\ ${ }^{1}$ Químico Industrial, Departamento de Química, Universidad de Los Andes, Carrera 1 № 18 A 12, Bogotá, Colombia, Imejia@ \\ uniandes.edu.co; ${ }^{2}$ Químico, Departamento de Química, Universidad de Los Andes, Carrera 1 № 18 A 12, Bogotá, Colombia, \\ rgomez@uniandes.edu.co
}

Rev. U.D.C.A Act. \& Div. Cient. 16(1): 215 - 222, 2013

\section{RESUMEN}

La especie vegetal Solanum pseudocapsicum L. (Solanacea), conocida comúnmente como "mirto", es una potencial fuente de estructuras funcionalizadas, que pueden ser empleadas en la obtención de fármacos esteroidales. En Colombia, no se han reportado estudios fitoquímicos de esta especie vegetal, que crece de forma silvestre en algunas regiones de nuestra geografía y, más aún, queda mucho por estudiar en cuanto al contenido de metabolitos secundarios, provenientes de esta planta. Tomando como base un método de extracción de carácter exploratorio, anteriormente evaluado para el género Solanum, se extrajeron de los frutos verdes y rojos de S. pseudocapsicum L. glicoalcaloides totales. Con el método utilizado, se obtuvo un rendimiento de $7,94 \mathrm{~g}$ y $2,48 \mathrm{~g}$ de glicoalcaloides totales por $\mathrm{kg}$ de fruto fresco para los frutos verdes y para los frutos rojos, respectivamente. El rendimiento obtenido es mayor al reportado en la India por Vijayan y colaboradores para esta especie vegetal. El precipitado obtenido de los frutos verdes fue hidrolizado y se pudo llegar a proponer la O-metilsolanocapsina, estructura dihidroderivada de la solanocapsina, como uno de los compuestos presentes.

Palabras clave: Mirto; glicoalcaloides; solanocapsina.

\section{SUMMARY}

The species Solanum pseudocapsicum L. known commonly in Colombia, as "mirto" is a potential source of highly functionalized structures that could be used as precursors of steroidal medicines. To the best of our knowledge phytochemical studies on this species have not yet been reported. Based on a previously described extraction method total glycoalkaloids from green and red fruits of $S$. pseudocapsicum L. were extracted. This method showed to be useful and $7.94 \mathrm{~g}$ and $2.48 \mathrm{~g}$ of glycoalkaloids per $\mathrm{kg}$ of fresh fruit in green and red fruits, respectively, were obtained. After hydrolysis of the initial precipitate, O-methyl solanocapsine, a structure derived from solanocpasine has been proposed as one of the components present in the extracts.

Key words: Myrthe; glycoalkaloids; solanocapsine.

\section{INTRODUCCIÓN}

Uno de los procesos químicos de mayor relevancia en la industria farmacéutica es la obtención de medicamentos con estructuras esteroidales, como hormonas sexuales y corticosteroides, que cumplen funciones de vital importancia en procesos metabólicos del hombre y los animales. Algunos de estos derivados son utilizados en tratamientos de desórdenes hormonales, metabólicos o como anticonceptivos orales, además se ha estudiado ampliamente la actividad de estas sustancias, como agentes anti-inflamatorios, diuréticos, anti-oxidantes, anti-virales, anti-microbianos y anti-tumorales (Usubillaga et al. 1997; Vijayan et al. 2002; 2004; Aliero et al. 2006; Dongre et al. 2007; Lien et al. 2012). Por tal motivo, se realizan constantes 
esfuerzos para descubrir nuevas fuentes de obtención de estructuras esteroidales básicas. Las especies del género Solanum son un recurso natural rico en alcaloides esteroidales, flavonoides y sus glicosidos, de los cuales, se ha sabido que poseen variada actividad biológica (Cornelius et al. 2010). A pesar que la literatura también reporta un amplio rango de variación en el contenido de glicoalcaloides de diferentes especies del género Solanum, muy pocos estudios reportan datos cuantitativos del contenido total de glicoalcaloides (Sanchez-Mata et al. 2010). La especie vegetal Solanum pseudocapsicum L. (Solanaceae), llamada popularmente en diferentes regiones de Colombia como "Mirto", "Grano de Oro", "Tomaticos" o "Espanta Pulgas" (Pérez-Arbeláez, 1996), se cultiva en viveros como planta ornamental o crece de forma silvestre en algunas regiones de Colombia. De acuerdo con Aliero et al. (2005) es un recurso útil y susceptible de ser usado en la obtención de estructuras esteroidales, que pueden ser modificadas químicamente para obtener fármacos o medicamentos y, según Jeyasankar et al. (2012), para la obtención de extractos que han mostrado cierta actividad biológica en larvas de insectos, lo cual, los hace potencialmente útiles en el control de plagas en agricultura, como plaguicidas naturales que, probablemente llegarían a reemplazar los pesticidas sintéticos, siendo un método netamente natural, no causaría ningún tipo de contaminación y sería completamente amigable con el medio ambiente.

La planta es un pequeño arbusto bien ramificado de tallos rectos y leñosos, con hojas alternas, sin estípulas, lanceoladas, con base atenuada. Las flores son pequeñas con cáliz persistente y solitarias, con segmentos agudos de pétalos blancos y estambres amarillos insertos en el tubo de la corola, nacen opuestas a las hojas. La planta se destaca por sus frutos en forma de baya globosa, con exocarpio liso de un color rojizo brillante o naranja rojizo de tonalidad más clara (García, 1975; Aliero et al. 2005). La morfología anatómica de las hojas, los tallos y los frutos maduros de esta especie vegetal, ha sido también investigada por métodos histológicos (Sanghvi et al. 2011).

Los alcaloides presentes en diferentes partes del arbusto de S. pseudocapsicum L. están representados, principalmente, por la solanocapsina, molécula esteroidal hexacíclica, con dos átomos de nitrógeno, uno de ellos extracíclico (Bruneton, 2001). Estudios realizados sobre plantas medicinales de la India han reportado en esta especie vegetal la presencia de una mezcla de esteroisómeros del 3-amino-16,23-dihidroxi22,26-epimino colestanos íntimamente asociados con la solanocapsina (Chakravarty et al. 1984; Dongre et al. 2007); en Sur África, se identificaron 41 compuestos volátiles, entre los que se destacan ácidos grasos, terpenoides y aldehídos, que fueron obtenidos por hidrodestilación (Aliero et al. 2007) y otros 25 tipos de compuestos diferentes, entre los que predominan hidrocarburos, alcoholes y derivados de ácidos carboxílicos, entre otros (Aliero et al. 2005). De otra parte, usando como disolvente una mezcla 1:1 metanol-agua y empleando un equipo Soxhlet, se extrajeron alcaloides totales en los frutos verdes de S. pseudocapsicum, con un rendimiento de 0,533\% (Vijayan et al. 2002) y 0,023\%, en frutos maduros (Vijayan et al. 2004). Varios alcaloides esteroidales, entre los que se incluyen solanocapsina, solacasina, solacapina, episolacapina, isosolacapina y O-metil-isosolanocapsina, han sido aislados de hojas, de frutos maduros, de tallos y de raíces de esta especie vegetal (Vijayan et al. 2004; Aliero et al. 2007; Dongre et al. 2007).

El presente estudio se realizó con el objetivo de determinar los alcaloides totales de la pulpa y de las semillas de los frutos verdes y rojos de S. pseudocapsicum L., iniciando así, a nivel exploratorio, las caracterizaciones de los metabolitos secundarios de esta especie vegetal.

\section{MATERIALES Y MÉTODOS}

Los alcaloides totales de la pulpa y de las semillas de los frutos verdes y rojos de S. pseudocapsicum L., se extrajeron teniendo como base un método de extracción, evaluado anteriormente con otras especies vegetales del género Solanum (Moreira et al. 1980), los cuales, fueron caracterizados mediante pruebas fitoquímicas clásicas, como Dragendorff y Wagner, específicas para alcaloides; los ensayos de Liebermann-Burchard y Salkowski, para soportar la presencia de estructuras esteroidales glicosídicas; la reacción de Felhing, para glucósidos (Bilbao, 1997) y, finalmente, la reacción de espuma, para saponinas y sapogeninas (Domínguez, 1979).

Los espectros UV-VIS de los precipitados fueron disueltos en etanol anhidro y registrado en un espectrofotómetro Marca Thermo Spectronic Modelo Genesys 2. Los espectros IR preparados en pastillas de $\mathrm{KBr}$ fueron obtenidos en un espectrofotómetro con transformada de Fourier ThermoNicolet Modelo NEXUS FT-IR. Los perfiles cromatográficos y los espectros de masas, se registraron con un cromatógrafo de gases, acoplado a detector selectivo de masas SHIMADZU Modelo GC-MS QP2010 S, con muestreador e inyector automático AOC 20i y fueron ensayados 4 tipos de columnas capilares diferentes: HP-5 (Crosslinked 5\% PH ME Siloxano) $30 \mathrm{~m} \times$ 0,32 I.D. x 0,25 $\mu \mathrm{m}$ df.; SHR5XLB SHIMADZU 30m x 0,25mm I.D. x 0,25 $\mu \mathrm{m}$ df.; HP-20M (Carbowax 20M) $25 \mathrm{~m}$ x 0,2mm I.D. x 0,2 $\mathrm{m}$ df., y HP-1701 (14\% CNPRPH ME Siloxano) $30 \mathrm{~m}$ x 0,32mm I.D. x $0.25 \mu \mathrm{m}$ df., para encontrar experimentalmente la mejor elución de los compuestos.

Material Vegetal: Se colectaron aleatoriamente frutos globosos sanos de S. pseudocapsicum L. de color verde y de color rojo, en tamaños aproximadamente iguales de diferentes arbustos 
y fueron caracterizados, siguiendo la metodología AOAC $7.003 / 84,930.15 / 90$, adaptado para \% de sustancias volátiles y la metodología AOAC 7.009/84,942.05/90, adaptado para \% de cenizas (Bernal, 1998). El lugar de recolección fue el departamento de Cundinamarca (Colombia), inmediaciones de la laguna de Fúquene, hacia el municipio de Susa, a una altura comprendida entre 2300 y 2400 msnm., ubicado en las coordenadas 5²7' de latitud norte y 7349' de longitud oeste, con una temperatura media anual cercana a los $14^{\circ} \mathrm{C}$, piso térmico frío. Un espécimen del material vegetal recolectado fue taxonómicamente identificado, autenticado y certificado en el Herbario Nacional Colombiano de la Universidad Nacional de Bogotá, quedando una muestra testigo, bajo el código 509380.

Preparación del material vegetal: Se retiraron las impurezas de los frutos lavándolos con suficiente agua potable; luego, fueron separadas manualmente las semillas de la pulpa, para enseguida secarlos en horno de convección con circulación de aire constante, a una temperatura de $50^{\circ} \mathrm{C}$, por un periodo de 96 horas. Posteriormente, se redujo el tamaño de partícula en un molino eléctrico, cuidando de no someter el material vegetal a sobrecalentamiento que pudiera degradar los compuestos y, a continuación, se tamizó a través de una malla No. 18. Así se obtuvieron muestras homogéneas, finamente divididas en tamaños de partícula $\leq 1,0 \mathrm{~mm}$, las cuales, se conservaron en recipientes de polipropileno cerrados, al abrigo de la humedad y la luz.

Extracción: $25 \mathrm{~g}$ de cada material vegetal preparado fue desengrasado durante $6 \mathrm{~h}$ en equipo Soxhlet, usando como disolvente éter etílico $(160 \mathrm{~mL})$; seguidamente, los compuestos polares fueron extraídos por reflujo contínuo durante 20h, con una solución etanólica de ácido acético al 10\% (150mL), produciendo extractos etanólicos crudos (EEC) que, al ser concentrados, permitieron obtener una pasta semisólida en un evaporador rotatorio, a una presión de $26 \mathrm{mbar}$. Se redisolvió en $150 \mathrm{~mL}$ de una solución acuosa de ácido acético al $10 \%$, calentando a $70^{\circ} \mathrm{C}$, durante $30 \mathrm{~min}$; después de filtrar al vacío, los líquidos obtenidos se calentaron de nuevo a $70^{\circ} \mathrm{C}$ y, posteriormente, con una solución de hidróxido de amonio al $28 \%$, se indujo la precipitación de los glicoalcaloides totales a pH 9,0 - 9,2, obteniendo una masa sólida de color ligeramente amarillo; ésta fue filtrada con una membrana de $0,45 \mu \mathrm{m}$, se secó y se pesó, para ser comparado con el porcentaje descrito en la literatura, para esta especie vegetal (Vijayan et al. 2004; 2002).

Precipitados obtenidos: Cada uno de los precipitados obtenidos de los extractos de las fracciones vegetales de pulpa y de semillas: pulpa de fruto rojo seco molido (PFRSM), semilla de fruto rojo seco molido (SFRSM), pulpa de fruto verde seco molido (PFVSM) y semilla de fruto verde seco molido (SFVSM) fueron evaluados cualitativamente con ensayos químicos clásicos específicos para alcaloides esteroidales $\mathrm{y}$, posteriormente, fueron analizados mediante técnicas físicas e instrumentales, como espectrofotometría ultravioleta visible (UV-VIS) e infrarrojo con transformada de Fourier (FT-IR).

A la mezcla de glicoalcaloides obtenidos de las semillas del fruto verde se les realizó una hidrólisis por reflujo continuo a $70^{\circ} \mathrm{C}$, con una solución de $\mathrm{HCl}$ al $7 \%$ en etanol, durante 4h, precipitando lentamente con hidróxido de amonio concentrado, extrayendo luego con porciones de $5 \mathrm{~mL}$ de diclorometano, separando las fases por centrifugación a 2000rpm y recuperando la fase medianamente polar. A la mezcla de alcaloides obtenida, se le aplicó un perfil de cromatografía de gases acoplada a espectrometría de masas (GC-MS). Los resultados experimentales de cuantificación gravimétrica permitieron comparar el contenido total de alcaloides del fruto rojo contra el fruto verde y contrastar el rendimiento contra el reportado en la literatura (Vijayan et al. 2004; 2002).

\section{RESULTADOS Y DISCUSIÓN}

En la tabla 1, se puede observar que el fruto verde presentó mayor porcentaje de residuo seco y de materia orgánica, condición que podría ser deseable para este estado vegetativo de los frutos de Solanum pseudocapsicum L. en el proceso de extracción de diferentes tipos de metabolitos, favorecido, además, por las condiciones bioecológicas del lugar de recolección de los frutos.

Usando método de reflujo continuo con etanol acidulado, previo tratamiento de desengrase con éter etílico en Soxhlet, se obtuvieron 12 extractos crudos: tres procedentes de

Tabla 1. Resultados del análisis próximo básico, que caracterizan los frutos recolectados.

\begin{tabular}{|l|c|c|}
\hline \multicolumn{1}{|c|}{ PARÁMETRO } & FRUTO ROJO & FRUTO VERDE \\
\hline Sustancias Volátiles a $105^{\circ} \mathrm{C}(\%)$ & 83,91 & 78,55 \\
\hline Cenizas (\%) & 2,24 & 2,89 \\
\hline Residuo Seco (\%) & 16,09 & 21,45 \\
\hline Materia Orgánica (\%) & 13,85 & 18,56 \\
\hline
\end{tabular}


semillas de fruto rojo, tres de semillas de fruto verde, tres de pulpas de fruto rojo y tres de pulpas de fruto verde de $S$. pseudocapsicum L., a los cuales, se le practicaron las pruebas de reconocimiento fitoquímicas cualitativas de Dragendorff, Wagner, Lieberman Burchard, Salkowski, la prueba de la espuma y la reacción de Fehling. Estas pruebas evidenciaron la presencia de glicoalcaloides esteroidales. De los extractos mencionados anteriormente, se obtuvieron precipitados que se caracterizaron cualitativamente y como se puede observar en la tabla 2, el análisis gravimétrico muestra que la mayor cantidad de glicoalcaloides totales en base seca se obtuvo, principalmente del fruto verde, donde se logró establecer que el rendimiento de alcaloides totales obtenido en el fruto fresco fue de $0,794 \%$, para frutos verdes y $0,248 \%$, para los frutos rojos, es decir, que de los frutos verdes se obtuvieron $7,94 \mathrm{~g}$ de glicoalcaloides totales por $\mathrm{kg}$, de fruto fresco y $2,48 \mathrm{~g}$ de glicoalcaloides totales por $\mathrm{kg}$ de fruto fresco, en el fruto rojo. Comparando entonces el resultado obtenido entre las fracciones vegetales es en las semillas de fruto verde, donde se halló la mayor concentración de alcaloides totales, que puede ser un indicativo que, en el estado vegetativo de los frutos rojos, se da una transformación o degradación de estos compuestos endógenos a otros metabolitos secundarios, que sería interesante estudiar. De otra parte, el resultado obtenido experimentalmente es mayor al reportado en la literatura para esta especie vegetal (Vijayan et al. 2004; 2002), que podría suponer una variación intraespecífica en esta especie vegetal. El método de extracción propuesto por Moreira (Moreira et al. 1980) había presentado ventajas significativas respecto al rendimiento de alcaloides totales obtenido, contra otros solventes y técnicas por él ensayadas en otras especies vegetales, siendo la extracción con etanol acidulado la principal diferencia frente al método usado por Vijayan et al. 2002).

Las bandas de máxima absorción observadas en el UV-VIS, para cada uno de los precipitados obtenidos, disueltos en metanol fueron respectivamente:

- PSFVSM: 210, 243, 285 y 312nm

- PSFRSM: 204, 285 y 318nm

- PFVSM: 204, 234, 285 y 315nm

- PFRSM: 204, 228, 285 y 318nm
Las bandas de absorción exhibidas en el UV-VIS hacia 204nm, $210 \mathrm{~nm}$, cercanas a $245 \mathrm{~nm}$ y $310 \mathrm{~nm}$, así como la resolución de bandas típicas en el infrarrojo hacia $3400 \mathrm{~cm}^{-1}, 1600 \mathrm{~cm}$ ${ }^{1}$ de grupos $(\mathrm{C}=\mathrm{N})$ y $1660 \mathrm{~cm}^{-1}$ y sumado a las pruebas fitoquímicas cualitativas, es evidencia típica de alcaloides esteroidales en los extractos obtenidos (Chakravarty et al. 1984; Domínguez, 1979).

El precipitado proveniente de los EEC de las semillas de fruto verde fue el que mostró mayor contenido de glicoalcaloides totales, por ello fue hidrolizado y posteriormente analizado con diferentes pruebas fitoquímicas cualitativas específicas y análisis instrumentales, como espectroscopía UV-VIS, FT-IR y cromatografía de gases, acoplada a espectrometría de masas GC-MS (Aliero et al. 2005;. 2006; Badami et al. 2005; Cipollini \& Levey 1997; Coelho et al. 1988; Eltayer et al. 1997; Jain \& Sharma, 1977; Laurila et al. 1999; Weissenberg, 2001).

Las pruebas fitoquímicas cualitativas realizadas revelaron claramente la presencia de compuestos esteroidales en el precipitado obtenido de la hidrólisis, que dieron verdaderamente positivas las pruebas de Liebermann Burchard y Salkowski y para compuestos alcaloídicos, los ensayos de Dragendorff y Wagner (Bilbao, 1997). Se deja en evidencia la presencia de núcleos espirostánicos o furostánicos, al dar positiva la prueba de la formación de espuma (Domínguez, 1979), se descarta la presencia de grupos glucosídicos al revelar una respuesta negativa el ensayo de Fehling, lo que indica que la hidrólisis fue exitosa y total. Al analizar por espectroscopía UV-VIS una fracción del compuesto hidrolizado disuelto en etanol, mostró definidamente absorciones máximas en 207 y 258nm; con base en lo anterior, se puede concluir que las absorciones mostradas del compuesto hidrolizado soportan la presencia de compuestos alcaloídicos esteroidales (Chakravarty et al. 1984; Domínguez, 1979).

El espectro de infrarrojo del producto hidrolizado, superpuesto con el espectro IR del precipitado de partida (Gráfica 1), pone de manifiesto que después del proceso de hidrólisis se observan bandas de mejor resolución hacia

Tabla 2. Resultados del rendimiento de glicoalcaloides totales obtenido para los extractos etanólicos crudos de cada fracción vegetal evaluada.

\begin{tabular}{|c|c|c|c|}
\hline \multirow{2}{*}{ Estado del Fruto } & \multicolumn{2}{|c|}{ GLICOALCALOIDES TOTALES (\%) } \\
\cline { 2 - 3 } & \multicolumn{2}{|c|}{ Fracción Vegetal } & \multirow{2}{*}{ SEMILLA + PULPA } \\
\cline { 2 - 3 } & SEMILLA & PULPA & \\
\hline FRUTO ROJO & $1,28 \pm 0,13$ & $0,52 \pm 0,19$ & $1,79 \pm 0,08$ \\
\hline FRUTO VERDE & $3,23 \pm 0,11$ & $1,05 \pm 0,23$ & $4,28 \pm 0,27$ \\
\hline
\end{tabular}




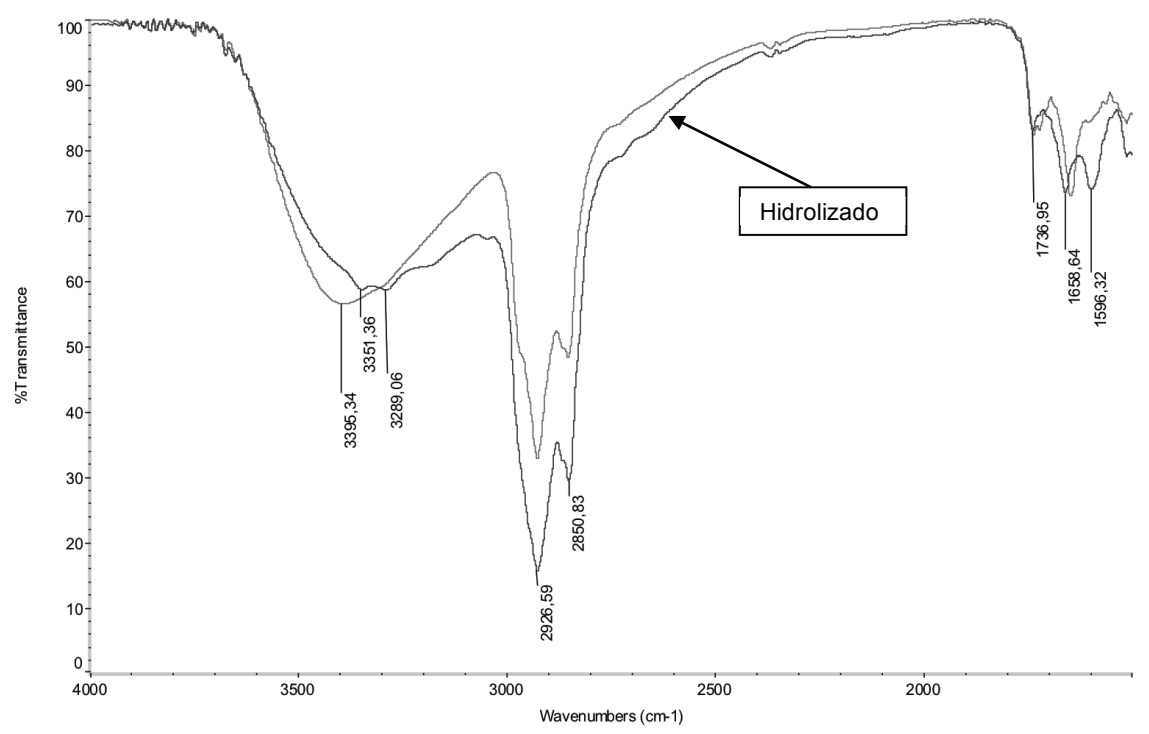

Gráfica 1. Espectro FT-IR comparativo entre el precipitado obtenido del extracto etanólico crudo de las semillas del fruto verde seco y molido y su fracción hidrolizada.

$3400 \mathrm{~cm}^{-1}, 1600 \mathrm{~cm}^{-1}$ de grupos $(\mathrm{C}=\mathrm{N})$ y $1660 \mathrm{~cm}^{-1}$, típicas de un alcaloide esteroidal obtenido de extractos alcohólicos de S. pseudocapsicum (Mitscher et al. 1976; Coll et al. 1984).

No se obtuvieron resultados satisfactorios ensayando las columnas capilares: HP-5; SHR5XLB y HP-20M, ya que no mostraron elución de compuestos que proporcionaran información representativa que confirmaran la presencia de estructuras esteroidales. El mejor resultado cromatográfico (Gráfica 2), se obtuvo para el precipitado hidrolizado de las semillas verdes usando la columna capilar HP-1701 (14\% CNPRPH Me Siloxano) $30 \mathrm{~m}$ x 0,32mm I.D. x 0,25 $\mu \mathrm{m}$ df., en las siguientes condiciones: temperatura del inyector $27^{\circ} \mathrm{C}$, relación split $10: 1$, gas de arrastre helio, control de flujo velocidad lineal $46,5 \mathrm{~cm} / \mathrm{s}$, flujo en la columna $1,5 \mathrm{~mL} /$ min, temperatura inicial en el horno $150^{\circ} \mathrm{C}$, velocidad de calentamiento $15^{\circ} \mathrm{C} / \mathrm{min}$, temperatura final $270^{\circ} \mathrm{C}$ durante $40 \mathrm{~min}$, temperatura de la Interfase $270^{\circ} \mathrm{C}$, temperatura de la fuente de iones $240^{\circ} \mathrm{C}$, voltaje de ionización: $70 \mathrm{~V}$, corriente de emisión: $35 \mu \mathrm{A}$, voltaje del detector: $0,89 \mathrm{kV}$.

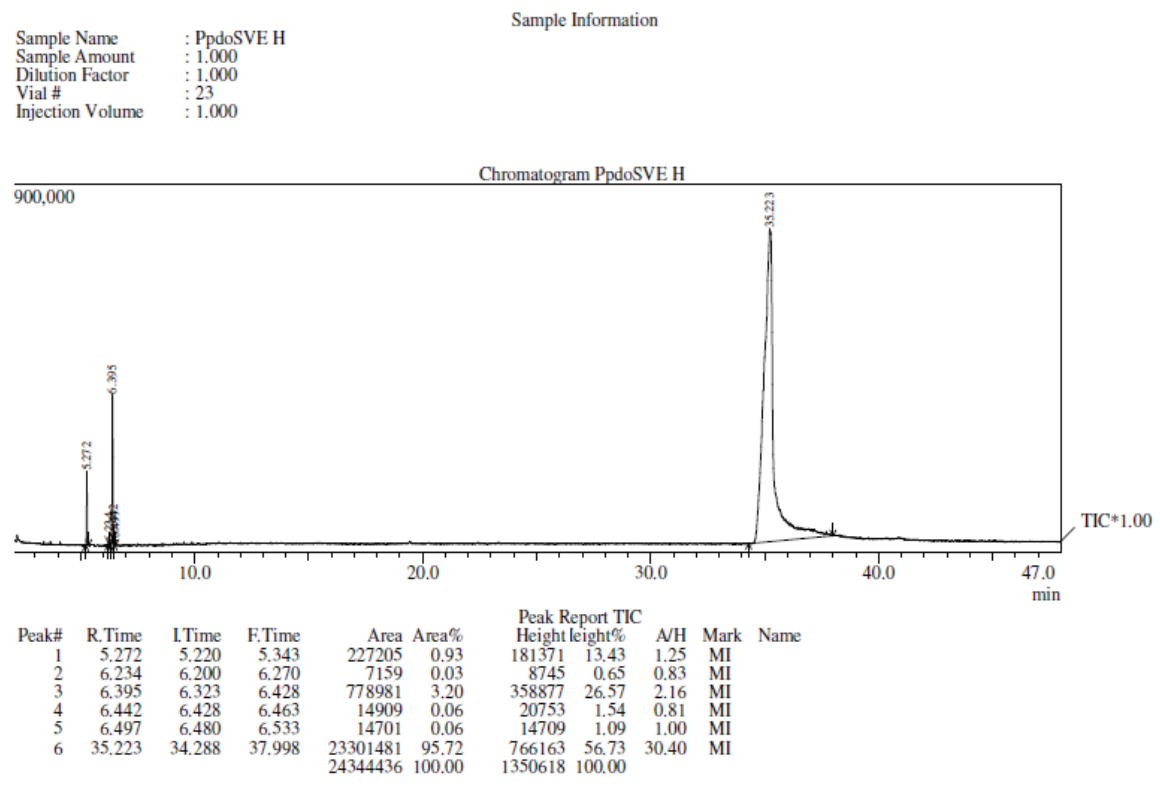

Gráfica 2. Perfil cromatográfico del precipitado hidrolizado, obtenido de las semillas del fruto verde seco y molido. 
El espectro de masas de la señal que aparece para el tiempo de retención (Tr) de 35,22min (Gráfica 3) mostró que varios de los posibles fragmentos de la muestra hidrolizada, entre ellos m/z 70, m/z 112, m/z 171, m/z 144, m/z 255, m/z 301, $\mathrm{m} / \mathrm{z} 314$ y $\mathrm{m} / \mathrm{z}$ 329, coinciden con señales típicas $\mathrm{m} / \mathrm{z}$ de núcleos esteroidales. Con base en las estructuras amino

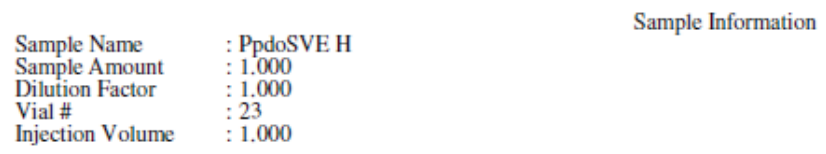

Spectrum

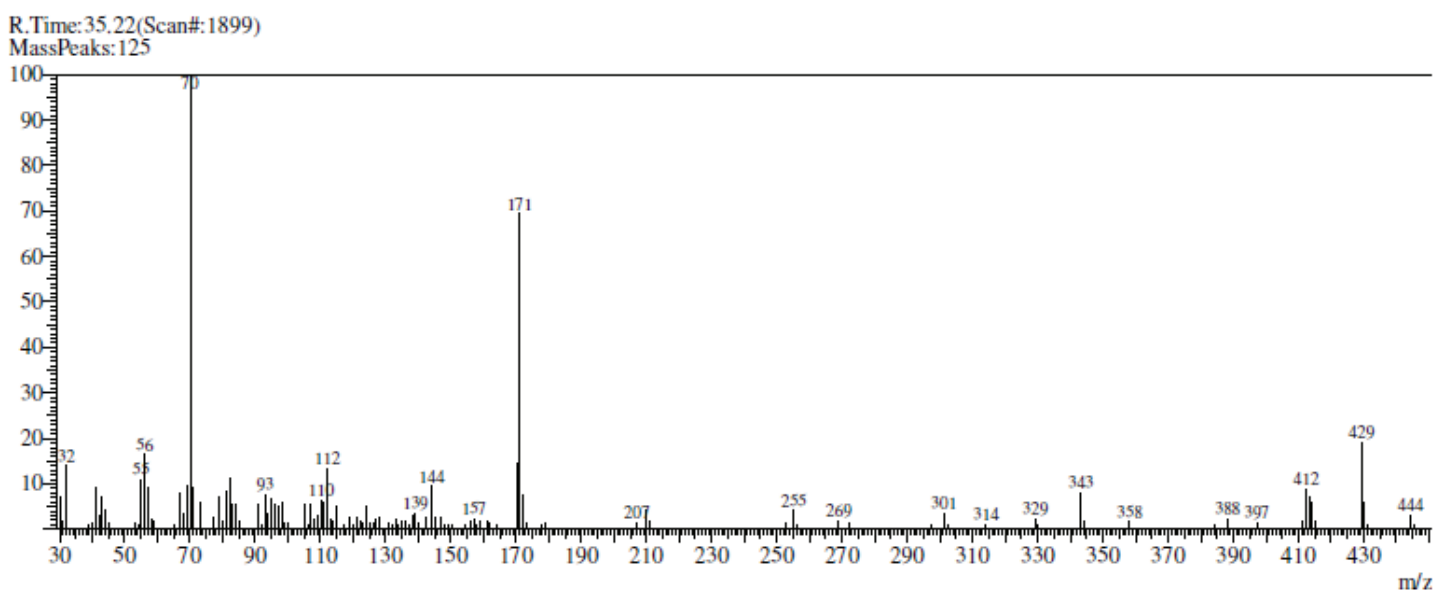

Gráfica 3. Espectro de masas obtenido en el tiempo de retención 35,22 min. del precipitado hidrolizado, obtenido de las semillas del fruto verde seco y molido.

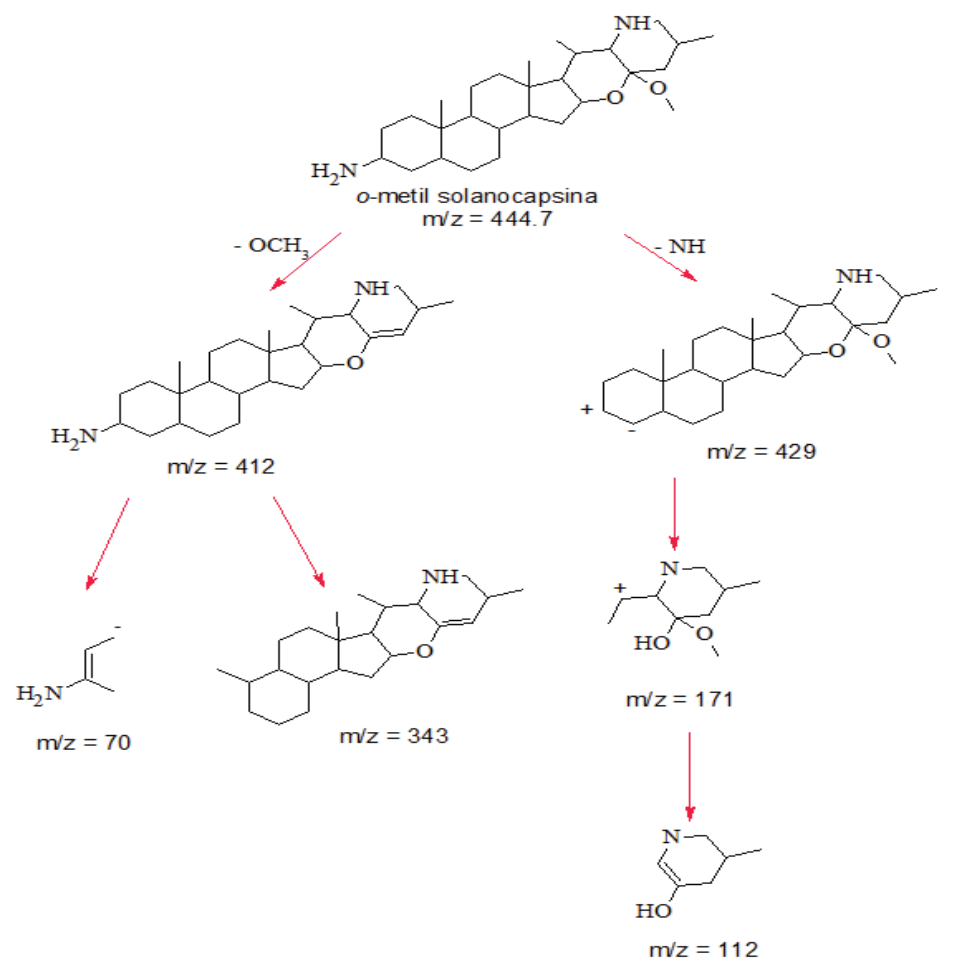

Figura 1. Fragmentos MS-IE propuestos para el tiempo de retención 35,22min. 
espirosolanas, estudiadas por Chakravarty et. al (1984), se propone para el perfil de masas, obtenido en Tr 35,22 min, el rompimiento, mostrado en la figura 1, que corresponde a la estructura de la solanocapsina, posiblemente la O-metil solanocapsina, donde el grupo metil esta unido por un oxígeno al carbono 22 y es similar a la estructura reportada por Dongre et al. (2007); así mismo, Coll et al. (1984) reportan, para esta estructura, los fragmentos $\mathrm{m} / \mathrm{z} 171$, $\mathrm{m} / \mathrm{z} 112$ y m/z 70, derivados del rompimiento de los anillos $\mathrm{E} / \mathrm{F}$ de la estructura esteroidal, similar a los fragmentos observados por Mitscher (Mitscher et al. 1976), en su orden $\mathrm{m} / \mathrm{z} 444, \mathrm{~m} / \mathrm{z} 429, \mathrm{~m} / \mathrm{z} 412, \mathrm{~m} / \mathrm{z} 343, \mathrm{~m} / \mathrm{z} 171, \mathrm{~m} / \mathrm{z} 144, \mathrm{~m} / \mathrm{z}$ $139, \mathrm{~m} / \mathrm{z} 112, \mathrm{~m} / \mathrm{z} 111, \mathrm{~m} / \mathrm{z} 82, \mathrm{~m} / \mathrm{z} 70$ y m/z 56, coherente con los fragmentos de masa obtenidos en este trabajo, para el precipitado hidrolizado de la semilla del fruto verde.

Conflictos de intereses: Este manuscrito fue preparado y revisado con la participación de todos los autores, quienes declaramos que no existe ningún conflicto de intereses que ponga en riesgo la validez de los resultados presentados. Financiación: Este estudio se desarrolló con el apoyo logístico del Departamento de Química de la Universidad de Los Andes y la Universidad de Ciencias Aplicadas y Ambientales U.D.C.A.

\section{BIBLIOGRAFÍA}

1. ALIERO, A.A.; ASEKUN, O.T.; GRIERSON, D.S.; AFOLAYAN, A.J. 2007. Volatile components from the roots of Solanum pseudocapsicum. J. Med. Food (Estados Unidos). 10(3):557-558.

2. ALIERO, A.A.; ASEKUN, O.T.; GRIERSON, D.S.; AFOLAYAN, A.J. 2006. Chemical composition of the hexane extract from the leaves of Solanum pseudocapsicum. Asian J. Plant. Sci. 5(6):10541056.

3. ALIERO, A.A.; GRIERSON, D.S.; AFOLAYAN, A.J. 2005. Chemical and nutrient characterization of Solanum pseudocapsicum berries. Afr. J. Biotechnol. (Kenia). 4(11):1300-1303.

4. BADAMI, S.; PRAKASH, O.; DONGRE, S.H.; SURESH, B. 2005. In vitro antioxidant properties of Solanum pseudocapsicum leaft extracts. India J. Pharmacol. (India). 37(4):251-252.

5. BERNAL, I. 1998. Análisis de alimentos. Ed. Guadalupe. (Colombia). 313p.
6. BILBAO, M. 1997. Análisis fitoquímico preliminar: química de productos naturales. Ed. Oficina Publicaciones U. del Quindío (Colombia). 187p.

7. BRUNETON, J. 2001. Plantas tóxicas: vegetales peligrosos para el hombre y los animales. Ed. Acribia. (España). 472p.

8. CHAKRAVARTY, A.K.; DAS, B.; ALI, E.; PAKRASHI, S.C. 1984. Studies on indian medicinal plants. Part 77. Structure and stereochemistry of some new steroidal alkaloids from and Solanum giganteum by nuclear magnetic resonance spectroscopy. J. Chemical Soc., Perkin Trans. 1 (Reino Unido). 1:467-474.

9. CIPOLLINI, M.L.; LEVEY, D. 1997. Antifungal activity of Solanum fruit glycoalkaloids: implications for frugivory and seed dispersal. Ecology (Estados Unidos). 98(3):799-809.

10. COELHO, R.M.; DE SOUZA, M.C.; SARRAGIOTTO, M.H. 1988. Steroidal alkaloid glycosides from Solanum orbignianum. Phytochem. (Paises Bajos). 49(3):893-897.

11. COLL, F; BASTERECHEA, M.; KUTSCHABSKY, L.; MOLA, D.L.; SCHREIBER, K.; ADAM, G. 1984. Aculeamine, a solanocapsine-type steroidal alkaloid from Solanum aculeatum. Phytochem. 23(4):883-885.

12. CORNELIUS, M.T.F.; DE CARVALHO, M.G.; DA SILVA, T.M.S.; ALVES, C.C.F.; SISTON, A.P.N.; ALVES, K.Z.; SANT'ANNA, C.M.R.; NETO, M.B.; EBERLIN, M.N.; BRAZ-FILHO, R. 2010. Other chemical constituents isolated from Solanum crinitium Lam. (Solanaceae). J. Braz. Chem. Soc. 21(12):2211-2219.

13. DONGRE, S.H.; BADAMI, S.H.; ASHOK, G.; RAVI, S.; KOTTANI, R. 2007. In vitro cytotoxic properties of O-methyl solanocapsine isolated from Solanum pseudocapsicum leaves. Indian J. Pharmacol. 39(4):208-209.

14. DOMÍNGUEZ, X. 1979. Métodos de investigación fitoquímica. Ed. Limusa (Méjico). 281p.

15. ELTAYER, E.A.; Al-ANSARI, A.S.; RODDICK, J.G. 1997. Changes in the steroidal alkaloid solasodine during development of Solanum nigrum and Solanum incanum. Phytochem. 46(3):489-494. 
16. GARCÍA, H. 1975. Flora medicinal de Colombia: botánica médica. Ed. Imprenta Nacional (Colombia). 107p.

17. JAIN, S.C.; SHARMA G.L. 1977. Studies on Solanum jasminoides for solasodine. Planta Medica (Alemania). 32:234-235.

18. JEYASANKAR, A.; PREMALATHA, S.; RANI, S.J. 2012. Bio-efficacy of Solanum pseudocapsicum L. (solanaceae) against black cutworm, Agrotis ipsilon Hufnagel (Lepidoptera: Noctuidae). J. Biol. Sci. (Pakistan). 12(3):174-179.

19. LAUIRILA, J.; LAAKSO, I.; VÄÄNÄNEN, T.; KURONEN, P.; HUOPALAHTI, R.; PEHU, E. 1999. Determination of solanidine and tomatidine type glycoalkaloid aglycons by gas chromatography/ mass spectrometry. J. Agr. Food Chem. (Estados Unidos). 47:2738-2742.

20. LIEN, E.; LIEN, L.; WANG, R.; WANG, J. 2012. Phytochemical analysis of medicinal plants with kidney protective activities. Chin. J. Integr. Med. (China). 18(10):790-800.

21. MITSCHER, L.A.; JUVARCAR, J.V.; BEAL, J.I. 1976. Solacasine, a new steroidal alkaloid from Solanum pseudocapsicum possessing antimicrobial activity. Cell. Molecular Life Sci., Experientia, Specialia. (Holanda). 32(4):415-416.

22. MOREIRA, E.A.; CECY, C.; NAKASHIMA, T.; CAVAZZANI, J.R.; GOMES, O.; KRAMBECK, R. 1980. Trabalho realizado do nucleo de pesquisa de produtos naturais do departamento de farmacia do setor de ciéncias da saúde da UFPR. VI Simposio de Plantas Medicinais do Brasil (Fortaleza, Brasil). p.114-126.

23. PÉREZ-ARBELÁEZ, E. 1996. Plantas útiles de Colombia. Ed. Fondo FEN (Colombia). p.709.
24. SANCHEZ-MATA, M.C.; YOKOYAMA, W.E.; HONG, Y.J.; PROHENS, J. 2010. $\alpha$-Solasonine and a-Solamargine contents of gboma (Solanum macrocarpon L.) and scarlet (Solanum aethiopicum L.) eggplants. J. Agric. Food Chem. 58:5502-5508.

25. SANGHVI, G.V.; KOYANI, R.D.; PATIL, V.S.; RAJPUT, K.S. 2011. Morpho-anatomy of Solanum pseudocapsicum. Rev. Bras. Farmacogn. 21(1):1115.

26. USUBILLAGA, A.; AZIZ, I.; TETTAMANZI, M.C.; WAIBEL, R.; ACHENBACH, H. 1997. Steroidal alkaloids from Solanum sycophanta. Phytochem. 44(3):537543.

27. VIJAYAN, P.; VIJAYARAJ, P.; SETTY, P.H.C.; HARIHARPURA, R.C.; GODAVARTHI, A.; BADAMI, S.; ARUMUGAM, D.S.; BHOJRAJ, S. 2004. The cytotoxic activity of the total alkaloids isolated from different parts of Solanum pseudocapsicum. Biol. Pharmac. B. (Japón). 27(4):528-530.

28. VIJAYAN, P.; VINOD KUMAR, S.; DHANARAJ, S.A.; BADAMI, S.; SURESH, B. 2002. In vitro cytotoxic and anti-tumor properties of the total alkaloid fraction of unripe fruits of Solanum pseudocapsicum. Pharmaceutical Biology (Reino Unido). 40(6):456-460.

29. WEISSENBERG, M. 2001. Isolation of solasodine and other steroidal alkaloids and sapogenins by direct hydrolysis-extraction of Solanum plants or glycosides therefrom. Phytochem. 58:501-508.

Recibido: Septiembre 27 de 2012

Aceptado: Marzo 13 de 2013 